\title{
Cerebral blood flow response to flavanol-rich cocoa in healthy elderly humans
}

\author{
Farzaneh A Sorond ${ }^{1,2}$ \\ Lewis A Lipsitz ${ }^{2,4}$ \\ Norman K Hollenberg ${ }^{3,5}$ \\ Naomi DL Fisher ${ }^{3}$ \\ 'Department of Neurology, Stroke \\ Division; ${ }^{2}$ Institute for Aging Research, \\ Hebrew SeniorLife, Boston, MA; \\ ${ }^{3}$ Department of Medicine, Endocrine- \\ Hypertension Division; ${ }^{4}$ Department \\ of Medicine, Gerontology, Beth Israel \\ Deaconess Medical Center, Boston, \\ MA, USA; ${ }^{5}$ Department of Radiology, \\ Brigham and Women's Hospital, \\ Boston, MA
}

\begin{abstract}
Background and Purpose: Cerebral ischemia is a common, morbid condition accompanied by cognitive decline. Recent reports on the vascular health benefits of flavanol-containing foods signify a promising approach to the treatment of cerebral ischemia. Our study was designed to investigate the effects of flavanol-rich cocoa (FRC) consumption on cerebral blood flow in older healthy volunteers.
\end{abstract}

Methods: We used transcranial Doppler (TCD) ultrasound to measure mean blood flow velocity (MFV) in the middle cerebral artery (MCA) in thirty-four healthy elderly volunteers $(72 \pm 6$ years) in response to the regular intake of FRC or flavanol-poor cocoa (FPC).

Results: In response to two weeks of FRC intake, MFV increased by $8 \% \pm 4 \%$ at one week $(p=0.01)$ and $10 \% \pm 4 \%(p=0.04)$ at two weeks. In response to one week of cocoa, significantly more subjects in the FRC as compared with the FPC group had an increase in their MFV $(\mathrm{p}<0.05)$.

Conclusions: In summary, we show that dietary intake of FRC is associated with a significant increase in cerebral blood flow velocity in the MCA as measured by TCD. Our data suggest a promising role for regular cocoa flavanol's consumption in the treatment of cerebrovascular ischemic syndromes, including dementias and stroke.

Keywords: cerebral blood flow, flavanol, cocoa, transcranial Doppler ultrasound

\section{Introduction}

Endothelial function is an important determinant of cardiovascular performance (Stefanadis et al 2000; London et al 2001; Perticone et al 2001). Endothelium-derived factors, most notably nitric oxide (NO), play a major role in the control of vascular biology, not only in the peripheral but also in the cerebral circulation. NO deficiency has been associated with the development of atherosclerosis and increased cardiovascular risk (for reviews: Busse and Fleming 1996; Gewaltig and Kojda 2002), and also with delayed vasospasm in subarachnoid hemorrhage (Pluta 2006) and cerebral hypoperfusion in ischemic syndromes (Zhang and Iadecola 1994; Iadecola et al 1997; Kielstein and Zoccali 2005). Since the discovery of nitric oxide synthase (NOS) in the brain and cerebral arteries (Iadecola 1993; Iadecola et al 1993), evidence is mounting to secure $\mathrm{NO}$ as a critical regulator of brain perfusion.

A number of epidemiological studies have shown that regular consumption of foods and beverages rich in flavanols is associated with a decreased risk of cardio- and cerebrovascular mortality (Knekt et al 1996; Commenges 2000; Galli 2002; Youdim 2002). In the context of human nutrition, flavanols occur in noteworthy amounts in specific foods and beverages, including some teas and red wines (Renaud and de Lorgeril 1992) and, especially, cocoa (Hammerstone et al 2000). Replenishing endothelium-derived NO reserves may be involved in the vasoprotective effects of flavanols. In vitro data show that cocoa protects the vascular endothelium by improving NO availability (Karim et al 2000; Heiss et al 2003). Our group has 
recently shown that dietary intake of flavanol-rich cocoa (FRC) was associated with peripheral vasodilation in healthy human subjects via activation of the NO system, providing a plausible mechanism for the vasoprotective effects of flavanol-rich foods (Fisher et al 2003). The work of others supports this concept (Heiss et al 2003). Moreover, we have also shown that FRC enhanced several measures of endothelial function to a greater degree among older healthy subjects, who have impaired endothelial function as compared to the younger healthy subjects (Fisher and Hollenberg 2006). Data from a recent pilot study using functional magnetic resonance imaging (fMRI) showed an increase in the blood oxygenation level-dependent (BOLD) signal intensity in response to a cognitive task following the regular consumption of moderate doses ( 5 days of $150 \mathrm{mg}$ ) of cocoa flavanols in 16 healthy young subjects (Francis et al 2006). However, to this date, there have not been any direct studies on the effects of FRC on cerebral blood flow in humans.

Our study was designed to investigate the acute and shortterm effects of FRC consumption on cerebral blood flow in older healthy volunteers. We used transcranial Doppler (TCD) ultrasound to: (1) assess cerebral blood flow responses following one week of dietary intervention with either FRC or a flavanol-poor cocoa (FPC) control; and to (2) characterize the acute and short-term cerebral blood flow response following 2 weeks of dietary FRC intake.

\section{Methods}

\section{Subjects}

Thirty-four healthy people aged $59-83$ years (mean $72 \pm 5.7$ years) volunteered to participate in this study. Thirteen of the 34 subjects participated in a 2 week study exploring the acute and steady state cerebral blood flow responses to dietary consumption of FRC. Twenty-one of the 34 subjects participated in a randomized, double-masked, placebo-controlled, parallel-arm study of the cerebral blood flow response to one week of daily FRC versus flavanol-poor cocoa (FPC) intake. Subjects were recruited from the Harvard Cooperative Program on Aging subject registry. All subjects were carefully screened with a medical history, physical examination, and electrocardiogram to exclude any acute or chronic medical conditions. Subjects were nonsmokers with normal blood pressure and without diabetes; they refrained from alcohol, caffeine and all chocolate intake for at least 12 hours. See Table 1 . The study was approved by the Partners Healthcare institutional review board, and followed institutional guidelines.
Table I Baseline subject characteristics

\begin{tabular}{lll}
\hline & $\begin{array}{l}\text { Time course of } \\
\text { MFV response }\end{array}$ & $\begin{array}{l}\text { Flavanol-rich vs } \\
\text { flavanol-poor cocoa }\end{array}$ \\
\hline $\mathrm{N}$ & 13 & $2 \mathrm{I}$ \\
M:F ratio & $5: 8$ & $1 . \mathrm{I}: \mathrm{I}$ \\
Age, $y$ & $72.5(4)$ & $72.2(6)$ \\
Mean arterial pressure & $83.9(13)$ & $74.3(24)$ \\
MCA baseline MFV, cm/s & $47.0(19)$ & $54.2(10)$ \\
\hline
\end{tabular}

Notes: All values are mean (SD).

Abbreviations: MCA, middle cerebral artery; MFV, mean blood flow velocity.

\section{Experimental protocol}

\section{Instrumentation}

Subjects reported to the General Clinical Research Center at the Brigham and Women's Hospital in the postabsorptive state, $\geq 2$ hours after their last meal. Subjects were instrumented for heart rate (HR electrocardiogram) and beat-to-beat arterial pressure monitoring (ABP, Finapres, Ohmeda Monitoring Systems, Englewood, CO) as previously described (Sorond et al 2005). End-tidal $\mathrm{CO}_{2}$ was measured from nasal prongs, using a Vacumed $\mathrm{CO}_{2}$ Analyzer (Ventura, CA).

TCD ultrasonography (MultiDop X4, DWL-Transcranial Doppler Systems Inc., Sterling, VA) was used to measure changes in middle cerebral artery (MCA) blood flow velocity $(\mathrm{MFV})$ at rest and in response to changes in end-tidal $\mathrm{CO}_{2}$ (cerebral vasoreactivity, VR) before and after cocoa consumption. The same technician performed all the studies and the depths of insonation were recorded so they could be duplicated in the follow-up study. The MCA signal was identified according to the criteria of Aaslid and colleagues (1982) and recorded at a depth of 50 to $60 \mathrm{~mm}$. A MuellerMoll probe fixation device was used to stabilize the Doppler probe for the duration of the study. The envelope of the velocity waveform, derived from a fast-Fourier analysis of the Doppler frequency signal, was digitized at $500 \mathrm{~Hz}$, displayed simultaneously with the ABP, ECG, and end-tidal $\mathrm{CO}_{2}$ signals, and stored for later off-line analysis.

\section{Cerebral vasoreactivity(VR)}

Changes in MCA MFV were measured during alterations in end-tidal $\mathrm{CO}_{2}$ (Maeda et al 1994) to determine the effects of dietary intake of a FRC and FPC beverage on cerebral VR to $\mathrm{CO}_{2}$. In this technique cerebral MFVs in the MCA were measured continuously while subjects inspired a gas mixture of $5 \% \mathrm{CO}_{2}, 21 \% \mathrm{O}_{2}$, and balance nitrogen for 2 minutes and then mildly hyperventilated to an end-tidal $\mathrm{CO}_{2}$ of approximately $25 \mathrm{mmHg}$ for 2 minutes. To determine cerebrovascular reactivity using this technique, percent 
change in MCA blood flow velocities was plotted against end tidal $\mathrm{CO}_{2}$ in response to room air, breathing $5 \% \mathrm{CO}_{2}$ and mild hyperventilation. Cerebral VR was measured as the slope of this relationship and expressed as percent change in cerebral blood flow per $\mathrm{mmHg}$ change in end-tidal $\mathrm{CO}_{2}$. In addition, in order to reflect changes in ABP, we have also calculated $\mathrm{VR}$ as the slope of the relationship between cerebrovascular conductance $(\mathrm{CVC}=\mathrm{MFV} / \mathrm{ABP})$ and end-tidal $\mathrm{CO}_{2}$. This is referred to as $\mathrm{VR}_{\mathrm{cvc}}$.

Time course of cerebral blood flow response

Thirteen of the subjects ( 8 female and 5 male, $72.5 \pm 4$ years) were enrolled in the time course study to investigate the effect of acute and short-term intake of FRC on cerebral blood flow velocity in the MCA. The FRC (Cocoapro ${ }^{\mathrm{TM}}$, Mars, Incorporated) beverage used in this study was a dairybased cocoa drink formulated to deliver $900 \mathrm{mg}$ of cocoa flavanols daily, in divided doses. The drink packet was a dry blend that was reconstituted with water just prior to consumption. The compositional details of this drink (and the FPC drink) can be found in Table 2. On the first day of the study, MCA MFV measurements were taken prior to the consumption of the beverage and the again at 2, 4, 6 and 8 hours after ingestion of one serving of FRC (450 mg flavanols). Baseline study was done before breakfast (fasting) and before the first dose of the beverage. Lunch was served between readings at 4 and 6 hours. After this baseline study, volunteers were provided with the appropriate number of drink packets and asked to consume 2 packets

Table 2 Nutritional content of the study beverages

\begin{tabular}{lll}
\hline & $\begin{array}{l}\text { Flavanol-rich } \\
\text { cocoa (FRC) }\end{array}$ & $\begin{array}{l}\text { Flavanol-poor } \\
\text { cocoa (FPC) }\end{array}$ \\
\hline Cocoa flavanols, mg & 451.1 & 18.2 \\
Calories & $1 \mathrm{I} 8 . \mathrm{I}$ & $\mathrm{II} 7.2$ \\
Total fat, g & 1.4 & 1.5 \\
Cholesterol, mg & 4.4 & 4.9 \\
Total carbohydrates, g & 17.1 & 16.5 \\
Dietary fiber, g & 3.0 & 3.9 \\
Sugars, g & 9.4 & 9.2 \\
Protein, g & 9.4 & 9.4 \\
Caffeine, mg & 18.3 & 21.2 \\
Theobromine, mg & 336.5 & 327.4 \\
Sodium, mg & 105.1 & 155.0 \\
Potassium, mg & 530.1 & 644.8 \\
Calcium, mg & 243.7 & 241.2 \\
Iron, mg & 1.9 & 2.9 \\
Phosphorous, mg & 280.2 & 265.4 \\
Magnesium, mg & 85.9 & 78.4 \\
Zinc, mg & 1.6 & 1.6 \\
Copper, mg & 0.4 & 0.4 \\
Manganese, mg & 0.6 & 0.6 \\
\hline
\end{tabular}

(900 mg flavanols daily) of the beverage daily for two weeks. Participants returned at 7 and 14 days for repeat MCA MFV studies at baseline and at 2, 4, 6, and 8 hours after one serving (450 mg cocoa flavanols) of FRC.

\section{Flavanol-rich versus flavanol-poor cocoa}

Twenty-one subjects (10 females and 11 males, $72.2 \pm$ 6 years) were randomized in accordance with a doublemasked, parallel-arm placebo-controlled study, designed to compare the effect of one week of dietary intake of FRC versus FPC on MCA blood flow velocity. The FRC and FPC drinks were formulated so that they were matched for calories, macronutrients, micronutrients, and alkaloids and were indistinguishable by taste and overall appearance (see Table 2). To ensure that the identity of the drinks remained unknown to the investigators and study participants, the drink packets were provided in individually wrapped and coded packets.

After assessing the resting cerebral blood flow velocity and cerebral vasoreactivity in the MCA territory, each subject was randomized to a group and asked to consume 2 packets of either the FRC (900 mg flavanols per day) or FPC (36 mg flavanols per day) for one week. Study participants returned for a follow-up visit at the same time of the day as the baseline study to minimize the effect of diurnal variations in $\mathrm{CBF}$.

\section{TCD data processing}

All data were displayed and digitized in real time at 500 $\mathrm{Hz}$ with commercially available data acquisition software (Windaq, Dataq Instruments). MFV and BP waveforms were re-sampled at $1 \mathrm{~Hz}$ using a MATLAB program. Beat-to-beat $\mathrm{R}-\mathrm{R}$ interval, ABP (expressed as mean arterial pressure) and MFV (expressed as mean flow velocity) were determined from the R wave of the ECG and the maximum and minimum of the arterial pressure or MFV waveforms.

Beat-to-beat values for ABP and ABP were averaged across each trial for each individual. Cerebrovascular resistance (CVR) was calculated as the ratio of ABP to MFV.

\section{Statistical analysis}

Absolute values in MCA, MFV, and CVR, as well as ABP were compared using repeated measures ANOVA. For the FRC versus FPC comparison, changes in MCA, VR, MFV, CVR, and ABP were compared between FRC and FPC using a Student's t-test. Chi-squared analysis was used to compare FRC versus FPC responders. Significance was set at $\mathrm{p}<0.05$. Data are expressed as mean $\pm \mathrm{SE}$. 


\section{Results}

\section{Time course of cerebral blood}

\section{flow response}

Two weeks of FRC intake had a consistent and statistically significant $(n=13 ; p=0.04)$ effect on peak cerebral blood flow responses. The peak and steady state cerebral blood flow response prior to and following one and two weeks of daily FRC consumption is summarized in Figure 1. At baseline, one serving of FRC drink (450 mg cocoa flavanols) resulted in an initial decrease in resting MCA MFV at 2 and 4 hours post consumption, which gradually returned to baseline at 6 and 8 hours. After one week of daily FRC consumption, a single serving of the FRC drink was again associated with an initial decrease in MFV at 2 and 4 hours, which subsequently increased to $2 \% \pm 2 \%(\mathrm{p}=0.14)$ at 6 hours and $8 \%$ $\pm 4 \%(\mathrm{p}=0.01)$ at 8 hours after ingestion. After two weeks of FRC ingestion, the cerebral blood flow response continued to increase, with a smaller initial decrease at 2 and 4 hours, followed by a small increase at 6 hours and a significant increase at 8 hours $(10 \% \pm 4 \%, \mathrm{p}=0.04)$ post FRC. Overall, MAP and CVR did not change significantly in response to two weeks of FRC intake.

\section{Flavanol-rich versus flavanol-poor cocoa}

Cerebrovascular hemodynamic responses following either one week of FRC or FPC consumption are summarized in Table 3. One week of either FRC or FPC ingestion did not have a significant effect on blood pressure, CVR, or cerebral vasoreactivity.

Despite variable individual blood flow response, 6/11 subjects had at least a $10 \%$ increase in MFV following the consumption of the FRC drink. In sharp contrast, only 1 in 10 volunteers consuming the FPC drink demonstrated a $10 \%$ or greater change in MFV ( $p<0.05$ by chi-squared analysis). Although the FRC group had a larger percent increase in their MFV $(54 \% \pm 30 \%$ on FRC and $16 \% \pm 2 \%$ on FPC), because of larger variability in the responses, these group differences did not reach statistical significance.

\section{Discussion}

To our knowledge, this is the first study to directly investigate the effect of both acute and short-term FRC consumption on cerebral blood flow. We show that two weeks of regular FRC intake, providing $900 \mathrm{mg}$ of cocoa flavanols daily, resulted in a significant increase in peak cerebral blood flow response in the MCA. In most subjects, there was an initial decrease of cerebral blood flow during the first 2-4 hours after intake of a single serving of FRC. This response was attenuated over time and is most likely related to the caffeine content of cocoa (Cameron et al 1990; Lunt et al 2004; Haase et al 2005). The mechanisms underlying flavanol-mediated increased cerebral blood flow responses are unknown. Given that the cerebral blood flow changes were not associated with significant changes in blood pressure, other regulatory mechanisms, such as metabolic factors may be involved. The enhanced cerebral blood flow response to FRC over two weeks is suggestive of an activated biochemical pathway where subsequent stimuli result in a potentiated response. Based on early in vitro data that showed NOS activation by flavanols (Karim et al 2000; Schroeter et al 2006) and our own work, which showed that FRC induced NO-dependent peripheral vasodilation in healthy humans (Fisher et al 2003), we propose that the cerebrovascular effects of FRC consumption are also NOdependent. Studies using specific NOS inhibitors, such as $\mathrm{N}(\mathrm{G})$-nitro-L- arginine methyl ester (L-NAME), will help to better define this pathway in the future.

Cocoa is known to be rich in theobromine, a methylxanthine, and to contain much lower amounts of its structurally close relative caffeine. Our flavanol-rich cocoa drink contained about $30 \mathrm{mg}$ caffeine and about $600 \mathrm{mg}$ theobromine; the FRC drink contained nearly the same levels of both these alkaloids per serving. Scant data are available regarding the biologic effects of theobromine, although generally it is considered a weaker stimulant and a milder diuretic than caffeine (Schroeder 1951; Dorfman and Jarvik 1970). The cerebral hemodynamic effects of theobromine have not been reported in humans. In rats caffeine resulted in greater reductions in $\mathrm{CBF}$ as compared with theobromine (Grome and Stefanovich 1986). On the other hand, the acute cerebrovascular effects of caffeine are better understood. Caffeine has consistently been shown to reduce cerebral blood flow significantly, averaging 23\%-30\% reduction with acute dosing (Cameron et al 1990; Field et al 2003; Liu et al 2004). It is likely, therefore, that any increase in cerebral blood flow measured acutely following FRC consumption must emerge upon a background of vasoconstriction induced by its methylxanthine ingredients. The early fall in CBF seen in the time course studies likely represents the effects of the methylxanthines, which are then overcome.

Interventions that improve cerebral blood flow may have therapeutic implications for dementia and stroke, and also for orthostatic hypotension. The promise of decreasing the decline in cognitive function seen with dementia syndromes through maintenance of cerebral blood flow is real. Data from a recent pilot study demonstrate that acute ingestion of 


\section{Cerebral blood flow response to cocoa}

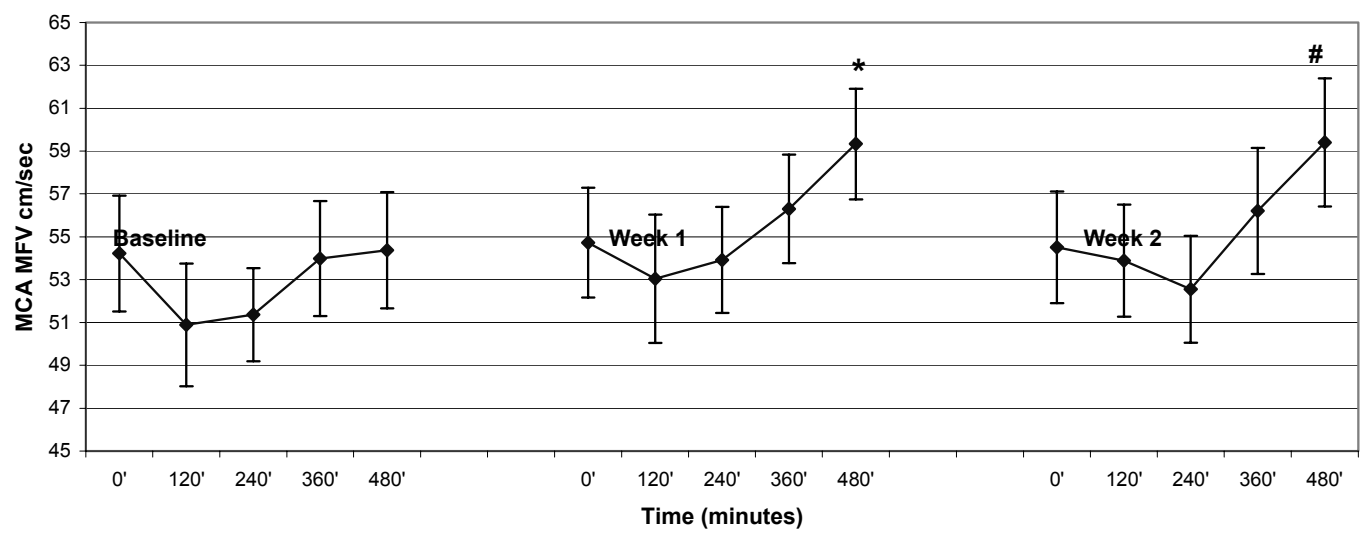

Blood pressure response to cocoa

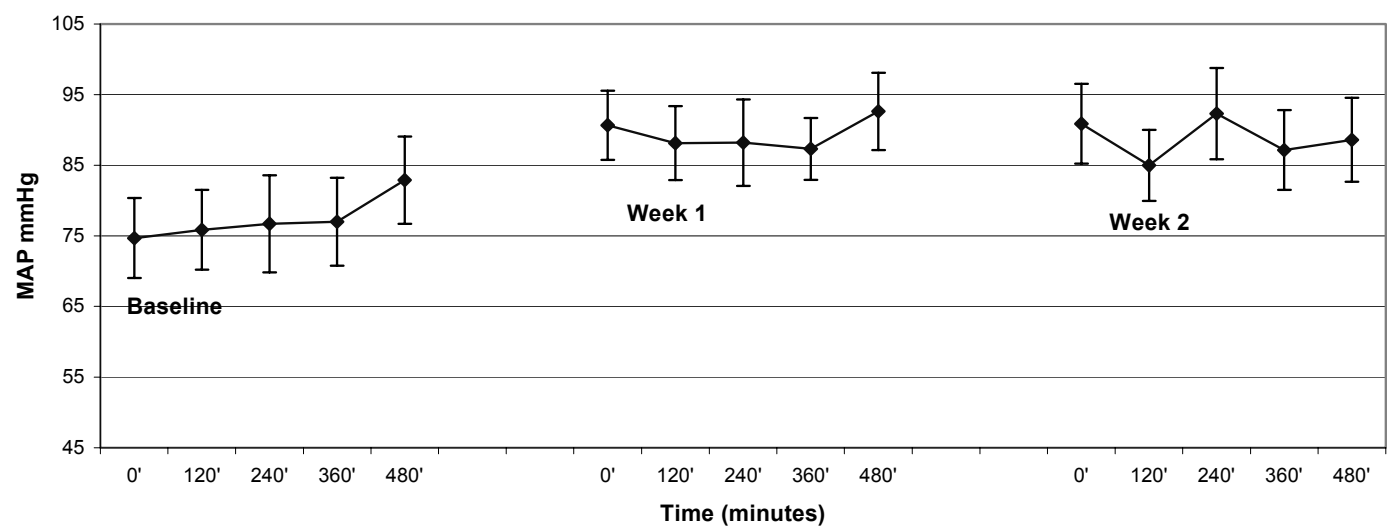

Cerebrovascular resistance response to cocoa

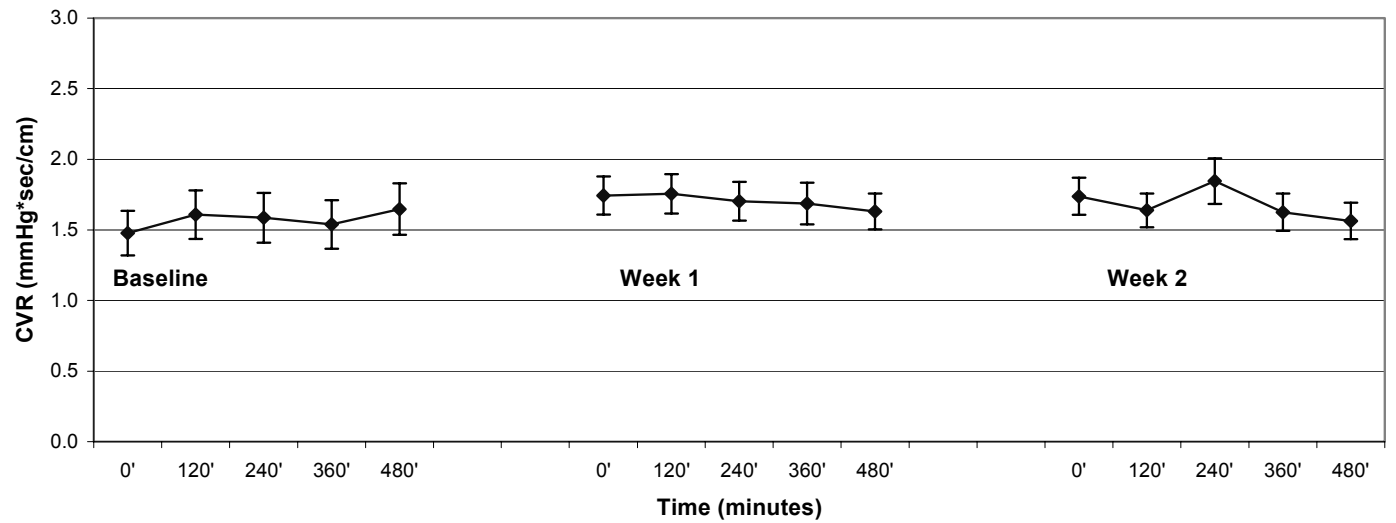

Figure I The cerebral blood flow (MCA MFV), blood pressure (MAP), and CVR response to FRC ingestions over 8 hours. Baseline denotes the response to the first dose. The same subjects were restudied at day 7 and 14 of the FRC-based dietary intervention. Note the sustained increase in the acute cerebral blood flow in response to FRC intake. Notes: *and \# denote points which show statistically significant changes from baseline.

Abbreviations: MCA, middle cerebral artery; MFV, mean blood flow velocity; MAP, mean arterial pressure; CVR, cerebrovascular resistance; FRC, flavanol-rich cocoa.

FRC could increase the cerebral blood flow to gray matter in young healthy volunteers (Francis et al 2006), supporting the promising role of cocoa flavanols for the treatment of dementias and strokes. There is compelling evidence from animal studies to show that reduced cerebral blood flow plays an initiating role in triggering cognitive dysfunction (Farkas et al 2002) and that improving cerebral blood flow can improve cognitive behavior (Farkas et al 2002; Basso 
Table 3 Cerebrovascular hemodynamics in response to one week of cocoa consumption

\begin{tabular}{|c|c|c|c|c|c|c|c|c|c|c|}
\hline & \multicolumn{5}{|c|}{ Baseline } & \multicolumn{5}{|c|}{ One week } \\
\hline & MAP & MFV & CVR & VR & VRcvc & MAP & MFV & CVR & VR & VRcvc \\
\hline FRC & $88(4)$ & $45(7)$ & $2.6(0.6)$ & I.3 (0.3) & $0.062(0.1)$ & $83(4)$ & $53(4)$ & I.7 (0.2) & I.4 (0.3) & $0.006(0.0)$ \\
\hline FPC & $80(4)$ & $49(5)$ & $1.9(0.3)$ & $1.6(0.4)$ & $0.004(0.0)$ & $78(4)$ & $50(5)$ & $2.1(0.5)$ & I.4 (0.2) & $0.001(0.0)$ \\
\hline
\end{tabular}

Notes: All values are mean (SE).

Abbreviations: MAP, mean arterial pressure; MCA, middle cerebral artery; MFV, mean blood flow velocity in the MCA; CVR, cerebrovascular resistance in the MCA (MAP/

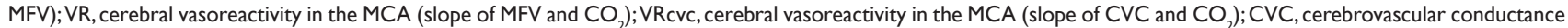
in the MCA (MFV/MAP); FRC, flavanol-rich cocoa; FPC, flavanol-poor cocoa.

et al 2005). There is a larger body of literature showing cerebral hypoperfusion is associated with impaired cognition (Ballard et al 1998; Puisieux et al 2001; Goldstein et al 2002; Ohtani et al 2003; Verghese et al 2003). We show here that the regular consumption of FRC significantly augments the peak cerebral blood flow response. The next step is to define the effects of this augmented peak blood flow response on ischemic syndromes as well as on cognitive performance, and to determine if it is necessary to achieve steady-state blood flow augmentation in order to improve cognitive and motor function.

Cerebral blood flow response to one week of daily FRC versus daily FPC intake demonstrated a favorable response to the FRC drink. Significantly more individuals responded to FRC by increasing their cerebral blood flow. However, a statistically significant difference was not achieved for the group steady state cerebral blood flow response. Knowing that FPC maintains $40 \%$ of the effect of the FPC on peripheral vascular responsiveness (Fisher et al 2003), and that our group MFV response of $16 \%$ to FPC was due to the individual response of one outlier, our results are consistent with our previous reports of the peripheral vascular response following flavanol-rich cocoa consumption.

Cognitive impairment due to cerebrovascular disease is a rapidly growing public health challenge. Recent epidemiological studies indicate that the total prevalence of vascular cognitive impairment (VCI), with or without dementia, could be as high as 3 million in the United States (Feldman et al 2003; Rockwood et al 2003), a number approaching that of Alzheimer's disease (AD). Despite the enormous health and financial costs associated with this cognitive syndrome (Fitzpatrick et al 2005; Sicras et al 2005), available treatments for VCI are limited to primary and secondary prevention of cerebrovascular disease. Recent reports on the vascular health benefits of flavanol-containing foods, added to our pilot data reported in this paper, signify a promising approach to the treatment of this cognitive syndrome.

In summary, our results reinforce and extend previously published pilot data in healthy young women (Francis et al 2006), and suggest that FRC intake directly increases cerebral blood flow in healthy elderly subjects. With this information, we can proceed to determine the effect of FRC consumption on cerebral ischemic syndromes including stroke, vasospasm, and vascular cognitive impairment.

\section{Study limitations}

We did not have a control for our two-week study subset of subjects. The ideal control for FRC would be a cocoa drink with equal amounts of methylxanthines, macronutrients, electrolytes, and calories, and devoid of flavanols. The technological and financial burdens to creating such a control are high; although no such control is currently available, we have made significant progress in designing this product for future studies. The FPC that was used in our one-week study contained some flavanols; this may well explain the partial peripheral and cerebrovascular responses compared with FRC. Moreover, we did not impose significant dietary restrictions on our subjects beyond avoidance of cocoa and caffeine-containing products, so the baseline flavanol intake of our subjects may have been quite varied. Background flavanol intake may be a factor that contributes to the magnitude of an individual's response, and should be evaluated in future work.

In addition, there a number of issues that can lead to enhanced variability in cerebral blood flow measurements using the TCD. The most important technical issue is the angle of insonation from one study to the other. In our time course study, by using a headband, which secured the TCD probe in one position through the course of the day, changes in angle of insonation between different measurements were prevented. However, in our study of FRC vs. FPC, the measurements were made two weeks apart. Although we recorded from the same depth and used the same technician for both studies, it is not possible to be sure that the angle was not slightly different. There is also the issue of time of day variations in cerebral blood flow velocity, which have an approximately 24 hour rhythm under constant conditions, suggesting regulation by a circadian oscillator. 
It is also important to note that transcranial Doppler ultrasound cannot provide an absolute measurement of cerebral blood flow, and BFV is assumed to represent blood flow through the MCA because it is assumed that the diameter of the MCA remains constant during manoeuvres that change cerebral blood flow. Using the thigh-cuff test, Newell and colleagues (1994) have shown that the absolute flow in the carotid artery showed a relative change and time course similar to velocity in the MCA. More recently, Serrador and colleagues (2000) reported that during $\mathrm{CO}_{2}$ manipulation or lower body negative pressure up to $40 \mathrm{mmHg}$ suction there were no changes detected in the diameter of MCA. Studies using a variety of techniques $\left({ }^{133} \mathrm{Xe}, \mathrm{SPECT}, \mathrm{MRI}\right)$ have confirmed that relative changes in $\mathrm{CBF}$ velocity are representative of changes in CBF (Bishop et al 1986; Sorteberg et al 1989; Dahl et al 1992; Vorstrup et al 1992; Larsen et al 1994, 1995; Sugimori et al 1995; Ulrich et al 1995; Clark et al 1996). Despite the lack of evidence about changes in the MCA diameter, this possibility cannot be eliminated and has to be acknowledged.

\section{Acknowledgment}

This work was supported in part with a research grant from Mars, Incorporated. Dr. Fisher is supported by RO3 AG023896 and Dr. Lipsitz by AG08812 and AG00439. There are no other conflicts of interest to report.

\section{References}

Aaslid R, Markwalder TM, Nornes H. 1982. Noninvasive transcranial Doppler ultrasound recording of flow velocity in basal cerebral arteries. J Neurosurg, 57:769-74.

Ballard C, Shaw F, McKeith I, et al. 1998. High prevalence of neurovascular instability in neurodegenerative dementias. Neurology, 51:1760-2.

Basso N, Paglia N, Cini R, et al. 2005. Effect of omapatrilat on the aging process of the normal rat. Cell Mol Biol (Noisy-le-grand), 51:557-64.

Bishop CC, Powell S, Rutt D, et al. 1986. Transcranial Doppler measurement of middle cerebral artery blood flow velocity: a validation study. Stroke, 17:913-5.

Busse R, Fleming I.1996. Endothelial dysfunction in atherosclerosis. J Vasc Res, 33:181-94.

Cameron OG, Modell JG, Hariharan M. 1990. Caffeine and human cerebral blood flow: a positron emission tomography study. Life Sci, 47:1141-6.

Clark JM, Skolnick BE, Gelfand R, et al. 1996. Relationship of 133Xe cerebral blood flow to middle cerebral arterial flow velocity in men at rest. J Cereb Blood Flow Metab, 16:1255-62.

Dahl A, Russell D, Nyberg-Hansen R, et al. 1992. A comparison of regional cerebral blood flow and middle cerebral artery blood flow velocities: simultaneous measurements in healthy subjects. J Cereb Blood Flow Metab, 12:1049-54.

Dorfman LJ, Jarvik ME. 1970. Comparative stimulant and diuretic actions of caffeine and theobromine in man. Clin Pharmacol Ther, 11:869-72.

Farkas E, de Wilde MC, Kiliaan AJ, et al. 2002. Chronic cerebral hypoperfusion-related neuropathologic changes and compromised cognitive status: window of treatment. Drugs Today (Barc), 38:365-76.
Feldman H, Levy AR, Hsiung GY, et al. 2003. A Canadian cohort study of cognitive impairment and related dementias (ACCORD): study methods and baseline results. Neuroepidemiology, 22:265-74.

Field AS, Laurienti PJ, Yen YF, et al. 2003. Dietary caffeine consumption and withdrawal: confounding variables in quantitative cerebral perfusion studies? Radiology, 227:129-35.

Fisher ND, Hollenberg NK. 2006. Aging and vascular responses to flavanolrich cocoa. J Hypertens, 24:1575-80.

Fisher ND, Hughes M, Gerhard-Herman M, et al. 2003. Flavanol-rich cocoa induces nitric-oxide-dependent vasodilation in healthy humans. J Hypertens, 21:2281-6.

Fitzpatrick AL, Kuller LH, Lopez OL, et al. 2005. Survival following dementia onset: Alzheimer's disease and vascular dementia. J Neurol Sci, 229-230: 43-9.

Francis ST, Head K, Morris PG, et al. 2006. The effect of flavanol-rich cocoa on the fMRI response to a cognitive task in healthy young people. J Cardiovasc Pharmacol, 47(Suppl 2):S215-20.

Gewaltig MT, Kojda G. 2002. Vasoprotection by nitric oxide: mechanisms and therapeutic potential. Cardiovasc Res, 55:250-60.

Goldstein IB, Bartzokis G, Guthrie D, et al. 2002. Ambulatory blood pressure and brain atrophy in the healthy elderly. Neurology, 59:713-9.

Grome JJ, Stefanovich V. 1986. Differential effects of methylxanthines on local cerebral blood flow and glucose utilization in the conscious rat. Naunyn Schmiedebergs Arch Pharmacol, 333:172-7.

Haase CG, Becka M, Kuhlmann J, et al. 2005. Influences of caffeine, acetazolamide and cognitive stimulation on cerebral blood flow velocities. Prog Neuropsychopharmacol Biol Psychiatry, 29:549-56.

Hammerstone JF, Lazarus SA, Schmitz HH. 2000. Procyanidin content and variation in some commonly consumed foods. $J$ Nutr, $130(8 \mathrm{~S}$ Suppl):2086S-92S.

Heiss C, Dejam A, Kleinbongard P, et al. 2003. Vascular effects of cocoa rich in flavan-3-ols. JAMA, 290:1030-1.

Iadecola C. 1993. Regulation of the cerebral microcirculation during neural activity: is nitric oxide the missing link? Trends Neurosci, 16:206-14.

Iadecola C, Zhang F, Casey R, et al. 1997. Delayed reduction of ischemic brain injury and neurological deficits in mice lacking the inducible nitric oxide synthase gene. J Neurosci, 17:9157-64.

Iadecola C, Zhang F, Xu X. 1993. Role of nitric oxide synthase-containing vascular nerves in cerebrovasodilation elicited from cerebellum. Am J Physiol, 264(4 Pt 2):R738-46.

Karim M, McCormick K, Kappagoda CT. 2000. Effects of cocoa extracts on endothelium-dependent relaxation. $J$ Nutr, 130(8S Suppl):2105S-8S.

Kielstein JT, Zoccali C. 2005. Asymmetric dimethylarginine: a cardiovascular risk factor and a uremic toxin coming of age? Am J Kidney Dis, 46:186-202.

Knekt P, Jarvinen R, Reunanen A, et al. 1996. Flavonoid intake and coronary mortality in Finland: a cohort study. BMJ, 312(7029):478-81.

Larsen FS, Olsen KS, Ejlersen E, et al. 1995. Cerebral blood flow autoregulation and transcranial Doppler sonography in patients with cirrhosis. Hepatology, 22:730-6.

Larsen FS, Olsen KS, Hansen BA, et al. 1994. Transcranial Doppler is valid for determination of the lower limit of cerebral blood flow autoregulation. Stroke, 25:1985-8.

Liu TT, Behzadi Y, Restom K, et al. 2004. Caffeine alters the temporal dynamics of the visual BOLD response. Neuroimage, 23:1402-13.

London GM, Blacher J, Pannier B, et al. 2001. Arterial wave reflections and survival in end-stage renal failure. Hypertension, 38:434-8.

Lunt MJ, Ragab S, Birch AA, et al. 2004. Comparison of caffeine-induced changes in cerebral blood flow and middle cerebral artery blood velocity shows that caffeine reduces middle cerebral artery diameter. Physiol Meas, 25:467-74.

Maeda H, Matsumoto M, Handa N, et al. 1994. Reactivity of cerebral blood flow to carbon dioxide in hypertensive patients: evaluation by the transcranial Doppler method. J Hypertens, 12:191-7.

Newell DW, Aaslid R, Lam A, et al. 1994. Comparison of flow and velocity during dynamic autoregulation testing in humans. Stroke, 25:793-7. 
Ohtani R, Tomimoto H, Kawasaki T, et al. 2003. Cerebral vasomotor reactivity to postural change is impaired in patients with cerebrovascular white matter lesions. $J$ Neurol, 250:412-7.

Perticone F, Ceravolo R, Pujia A, et al. 2001. Prognostic significance of endothelial dysfunction in hypertensive patients. Circulation, 104:191-6.

Pluta RM. 2006. Dysfunction of nitric oxide synthases as a cause and therapeutic target in delayed cerebral vasospasm after SAH. Neurol Res, 28:730-7.

Puisieux F, Monaca P, Deplanque D, et al. 2001. Relationship between leuko-araiosis and blood pressure variability in the elderly. Eur Neurol, 46:115-20.

Renaud S, de Lorgeril M. 1992. Wine, alcohol, platelets, and the French paradox for coronary heart disease. Lancet, 339(8808):1523-6.

Rockwood K, Davis H, MacKnight C, et al. 2003. The Consortium to Investigate Vascular Impairment of Cognition: methods and first findings. Can J Neurol Sci, 30:237-43.

Schroeder HA. 1951. Studies on congestive circulatory failure. IV. The effect of various diuretics on the excretion of water and chlorides. Circulation, 4:87-99.

Schroeter H, Heiss C, Balzer J, et al. 2006. (-)-Epicatechin mediates beneficial effects of flavanol-rich cocoa on vascular function in humans. Proc Natl Acad Sci USA, 103:1024-9.

Serrador JM, Picot PA, Rutt BK, et al. 2000. MRI measures of middle cerebral artery diameter in conscious humans during simulated orthostasis. Stroke, 31:1672-8.
Sicras A, Rejas J, Arco S, et al. 2005. Prevalence, resource utilization and costs of vascular dementia compared to Alzheimer's dementia in a population setting. Dement Geriatr Cogn Disord, 19(5-6):305-15.

Sorond FA, Khavari R, Serrador JM, et al. 2005. Regional cerebral autoregulation during orthostatic stress: age-related differences. J Gerontol A Biol Sci Med Sci, 60:1484-7.

Sorteberg W, Lindegaard KF, Rootwelt K, et al. 1989. Blood velocity and regional blood flow in defined cerebral artery systems. Acta Neurochir (Wien), 97(1-2):47-52.

Stefanadis C, Dernellis J, Tsiamis E, et al. 2000. Aortic stiffness as a risk factor for recurrent acute coronary events in patients with ischaemic heart disease. Eur Heart J, 21:390-6.

Sugimori H, Ibayashi S, Fujii K, et al. 1995. Can transcranial Doppler really detect reduced cerebral perfusion states? Stroke, 26:2053-60.

Ulrich PT, Becker T, Kempski OS. 1995. Correlation of cerebral blood flow and MCA flow velocity measured in healthy volunteers during acetazolamide and CO2 stimulation. J Neurol Sci, 129:120-30.

Verghese J, Lipton RB, Hall CB, et al. 2003. Low blood pressure and the risk of dementia in very old individuals. Neurology, 61:1667-72.

Vorstrup S, Zbornikova V, Sjoholm H, et al. 1992. CBF and transcranial Doppler sonography during vasodilatory stress tests in patients with common carotid artery occlusion. Neurol Res, 14:31-8.

Zhang F, Iadecola C. 1994. Reduction of focal cerebral ischemic damage by delayed treatment with nitric oxide donors. J Cereb Blood Flow Metab, 14:574-80. 